\title{
Paradoxical Herniation after Unilateral Decompressive Craniectomy: A Retrospective Analysis of Clinical Characteristics and Effectiveness of Therapeutic Measures
}

\author{
Huangyi $\mathrm{JI}^{1}$, Weiqiang $\mathrm{CHEN}^{1 *}$, Xiaohuan $\mathrm{YANG}^{2 *}$, Jingfang $\mathrm{GUO}^{1 *}$, Jin $\mathrm{WU}^{1}$, Mindong HUANG${ }^{3}$, Chuwei CAI ${ }^{4}$, \\ Yingming YANG ${ }^{1}$
}

\begin{abstract}
'Shantou University Medical College, First Affiliated Hospital, Department of Neurosurgery, Shantou, Guangdong, China ${ }^{2}$ Shantou University Medical College, First Affiliated Hospital, Department of Science and Education, Shantou, Guangdong, China

${ }^{3}$ The People's Hospital of Jieyang City, Department of Neurosurgery, Jieyang, Guangdong, China

${ }^{4}$ The Central Hospital of Shantou City, Department of Neurosurgery, Shantou, Guangdong, China

*These authors contributed equally to this work.
\end{abstract}

\section{ABSTRACT}

AIM: We aimed to investigate the importance of early diagnosis and proper management of paradoxical herniation based on the data of 13 patients who had 14 occurrences of paradoxical herniation.

MATERIAL and METHODS: The characteristics and the effectiveness of treatments of 13 patients with paradoxical herniation were reviewed and analyzed retrospectively.

RESULTS: Paradoxical herniation occurred in eight patients (61.54\%) during the postoperative 2 weeks and they presented with typical symptoms of brain herniation and a tense skin flap without sinking at the region of decompressive craniectomy. On the other hand, six patients developed paradoxical herniation in the postoperative period of 2 weeks to 2 months and presented with sinking skin flaps and delayed neurological deficits. Furthermore, all patients received emergency treatments, including sufficient hydration, clamping cerebrospinal fluid (CSF) drainage, and being placed in the Trendelenburg position. Six patients achieved full neurologic recovery after successful cranioplasty.

CONCLUSION: Intracranial hypotension causing paradoxical herniation can rapidly progress, especially along with CSF depletion. It is important for neurosurgeons to suspect paradoxical herniation in a subset of patients with large cranium defects and tense skin flap without sinking during the postoperative 2 weeks. Paradoxical herniation is rapidly reverted by improving CSF hydration, and performing early cranioplasty referred as the definitive treatment.

KEYWORDS: Paradoxical herniation, Decompressive craniectomy, Treatment

\section{INTRODUCTION}

$\mathrm{T}$ The patient with uncontrollable intracranial hypertension usually undergoes emergency decompressive craniectomy $(1,7,14)$. The decompressive craniectomy provided more space for the masses or edema of the brain
(14). However, it has been reported $(1,13,29,34)$ that the cranial conversion from a "closed box" to an "open box" affects the balance of pathophysiology, which brings risks. Paradoxical herniation, an unusual complication of cerebrospinal fluid (CSF) drainage in patients with large decompressive 
craniectomy, may result in coma or death because of delayed diagnosis or mismanagement. To date, the information on paradoxical herniation as procedural outcomes affecting patient survival and recovery is mostly based on sporadic case reports rather than multi-centered, randomized, controlled clinical trials $(1,13,29,34)$. Thus, the neurosurgeons need to do further clinical investigation. Here we present an analysis of the clinical characteristics and effectiveness of therapies based on the data of 13 patients who had 14 occurrences of paradoxical herniation which we have treated since 2007. Our study demonstrates the importance of early diagnosis and proper management of paradoxical herniation.

\section{MATERIAL and METHODS}

\section{Ethics Statement}

This retrospective study was approved by the ethics committee of First Affiliated Hospital, Shantou University Medical College (China). Written consent was given by the patients for their information to be stored in the hospital database and used for research.

\section{General Data}

We performed a retrospective review of 13 patients with paradoxical herniation identified in the databases of the neurosurgical departments of the first affiliated hospital, Shantou University Medical College, the people's hospital of Jieyang city and the central hospital of Shantou City. These cases, covering a period of 2007-2011, were supported with detailed clinical records including pre- and post-operative computed tomography (CT) scans and 6-month follow-up information. Patients' characteristics are summarized in Table I. The cohort comprised ten men and three women. The mean (SD) age of the patients was 57.9 years (ranged from 34 to 75 ). All of them underwent unilateral decompressive craniectomy because of deteriorated intracranial hypertension. There is no patient with bilateral decompressive craniectomy who presented paradoxical herniation in our hospital. In addition, five patients had severe brain injuries, four patients had ruptured aneurysms, and five patients had strokes. Postoperatively, 11 patients underwent lumbar puncture, seven patients underwent external ventriculostomy, six patients experienced continued lumbar CSF drainage, four patients underwent ventriculoperitoneal (VP) shunts, and one patient suffered CSF rhinorrhea (Figures 1A-D; 2A-H; 3A-I).

\section{Clinical Manifestation}

Paradoxical herniation occurred in eight cases during the 2 weeks postoperatively. Initial clinical presentations included tense skin flap without sinking at the region of decompressive craniectomy and typical symptoms of brain herniation, such as brainstem release signs, altered state of consciousness, and pupillary changes. Furthermore, six patients developed paradoxical herniation in the post-operative period of 2 weeks to 2 months presenting sinking skin flaps and delayed neurological deficits, such as persistent or orthostatic
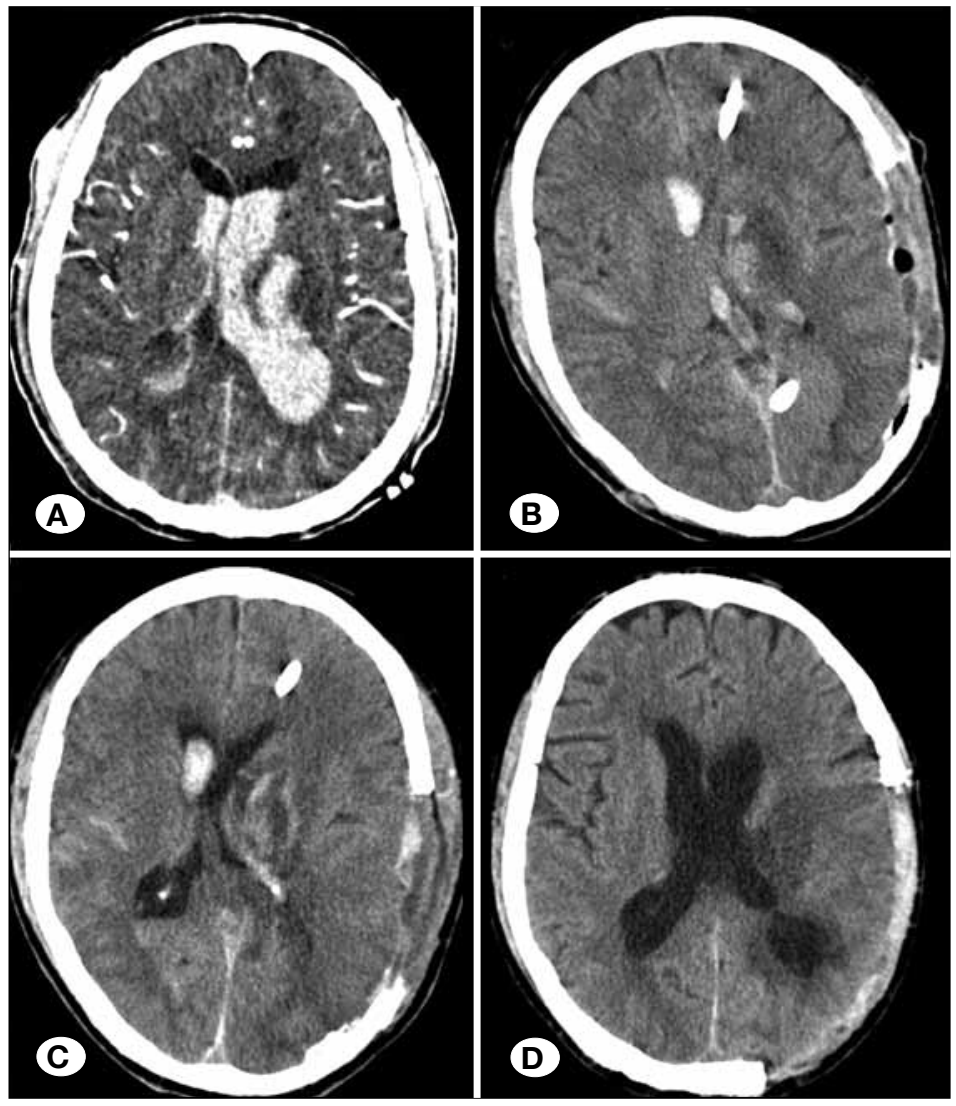

Figure 1: Patient 13 (shown in Table I) presented paradoxical herniation occurred during the postoperative 2 weeks. CT and CTA revealed left occipital hematoma rupturing into the ventricular system and no vascular malformations (A). On post-operative day (POD) 1, CT scan after decompressive craniectomy showed the volume of hematoma in the ventricle was markedly decreased with a slight shift of midline while the external drain of postoperative 24 hours was $280 \mathrm{ml}$ (B). On POD 5, the patient's neurological status deteriorated with ICP fluctuating between 1 and $3 \mathrm{~mm} \mathrm{Hg}$, the systolic blood pressure dropped to $85 \mathrm{~mm} \mathrm{Hg}$. The CT scan demonstrated a significant midline shift (C). After removing the external ventricular drainage, being placed in the Trendelenburg position and receiving sufficient hydration intravenously, CT scan on POD 14 showed the resolution of midline shift, mild ventricular expansion and significant reduction in the volume of hematoma (D). 


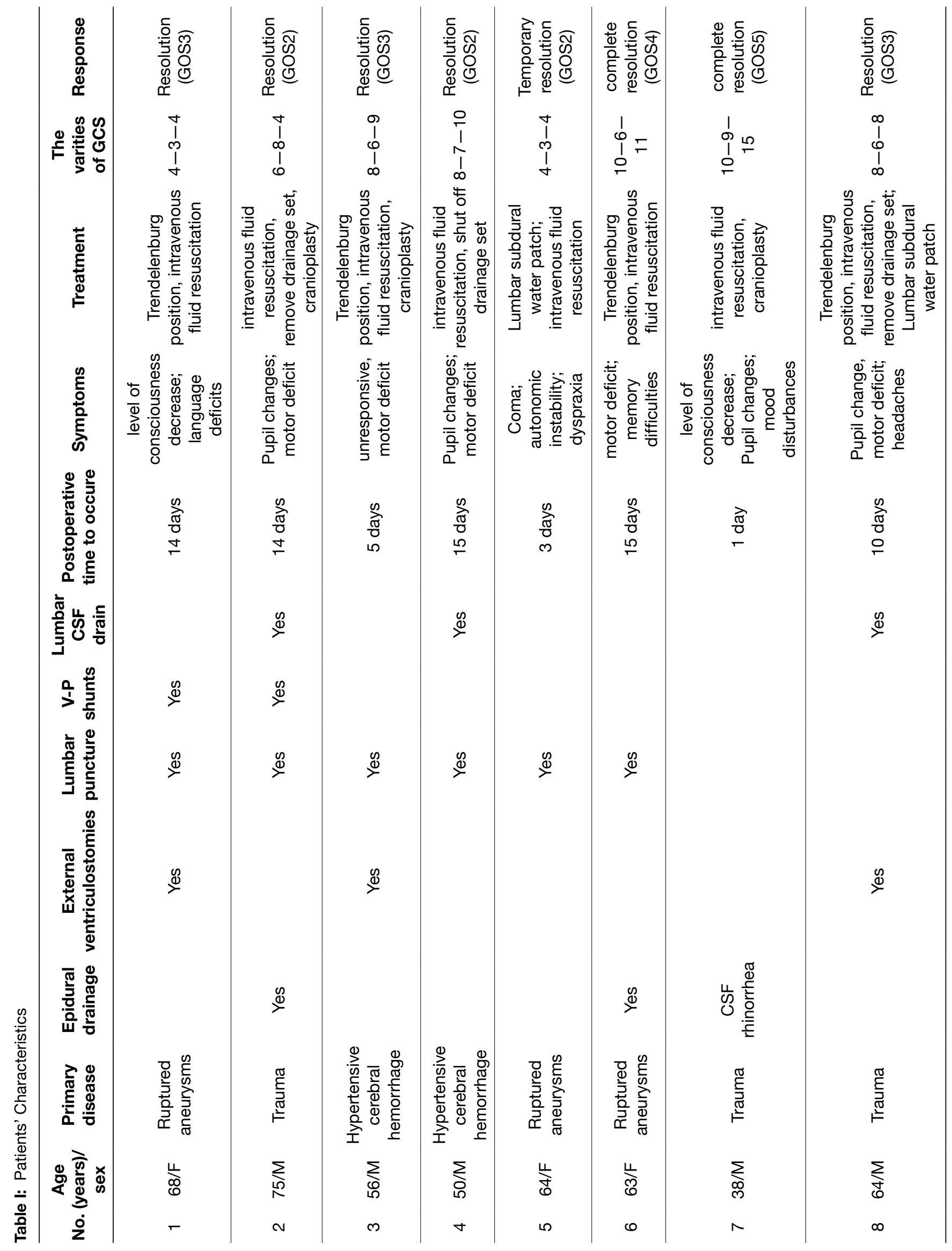




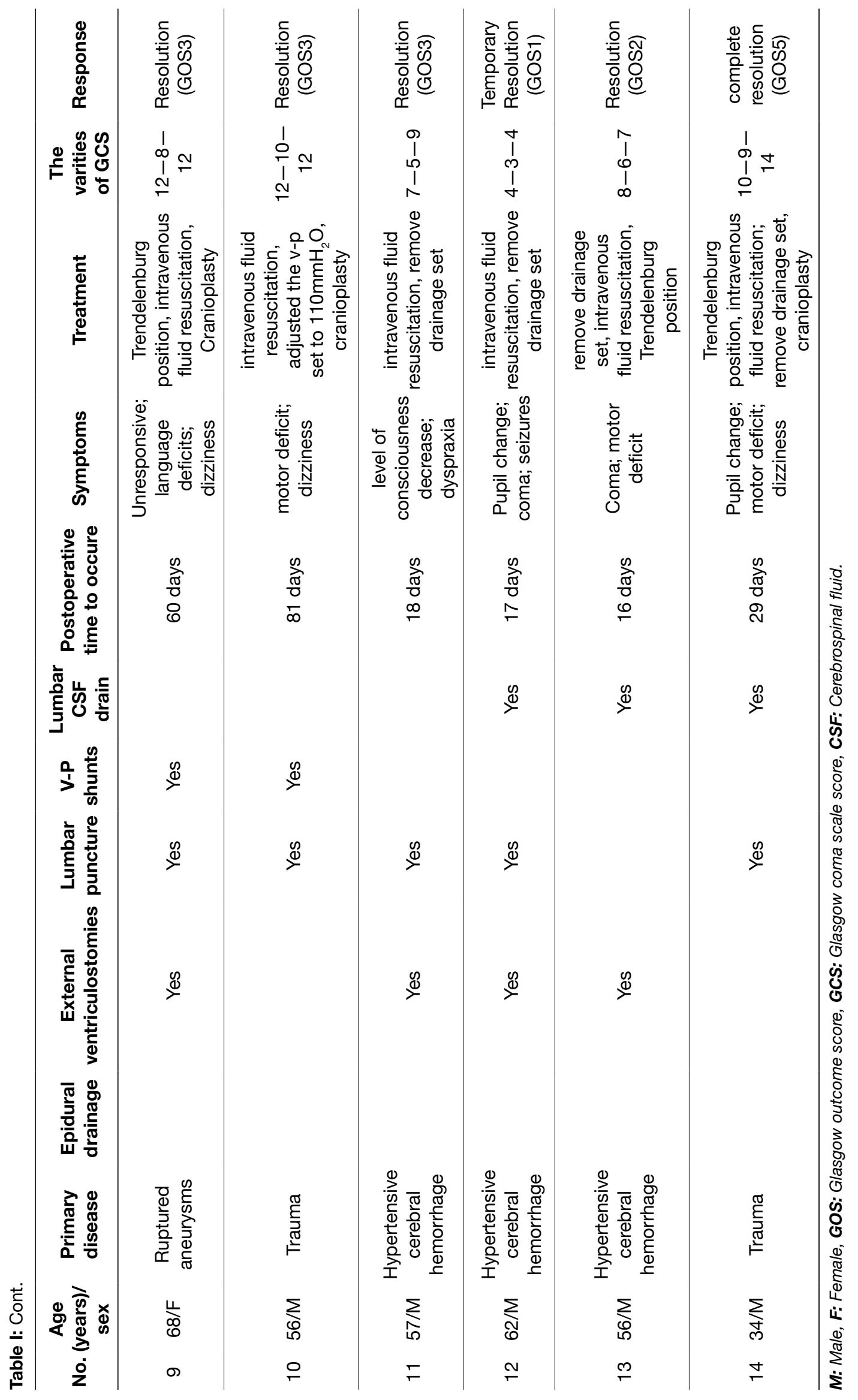


headaches, dizziness, mood disturbance, memory loss, autonomic instability and dyspraxia.

\section{Computed Tomography Features}

CT images can reveal the structural alterations suggesting intracranial hypotension: flattening of the pons along with shrinking or effacement of prepontine and perichiasmatic cisterns in eight patients, sinking skin flaps in six patients (as shown in Figure 2A-H), and subdural hygroma in seven patients. In addition, midline shifts in the direction opposite to the skull defects and brainstem compressions were shown in all patients.

\section{Intracranial Pressure (ICP)}

ICP was monitored in four patients (Codman $®$ intracranial pressure monitor), and on average, patients underwent 10 days of epidural ICP monitoring. The data showed that ICP ranged from -3 to $3 \mathrm{~cm} \mathrm{H}_{2} \mathrm{O}$, and the variations of the blood pressure were $72-81 / 35-46 \mathrm{mmHg}$ in patients with paradoxical herniation. 13 patients underwent lumbar puncture presented low ICP fluctuating between 3 and $5 \mathrm{~cm} \mathrm{H}_{2} \mathrm{O}$. Due to such low ICP, an extraction was done using a syringe along the puncture tube of the CSF drain.
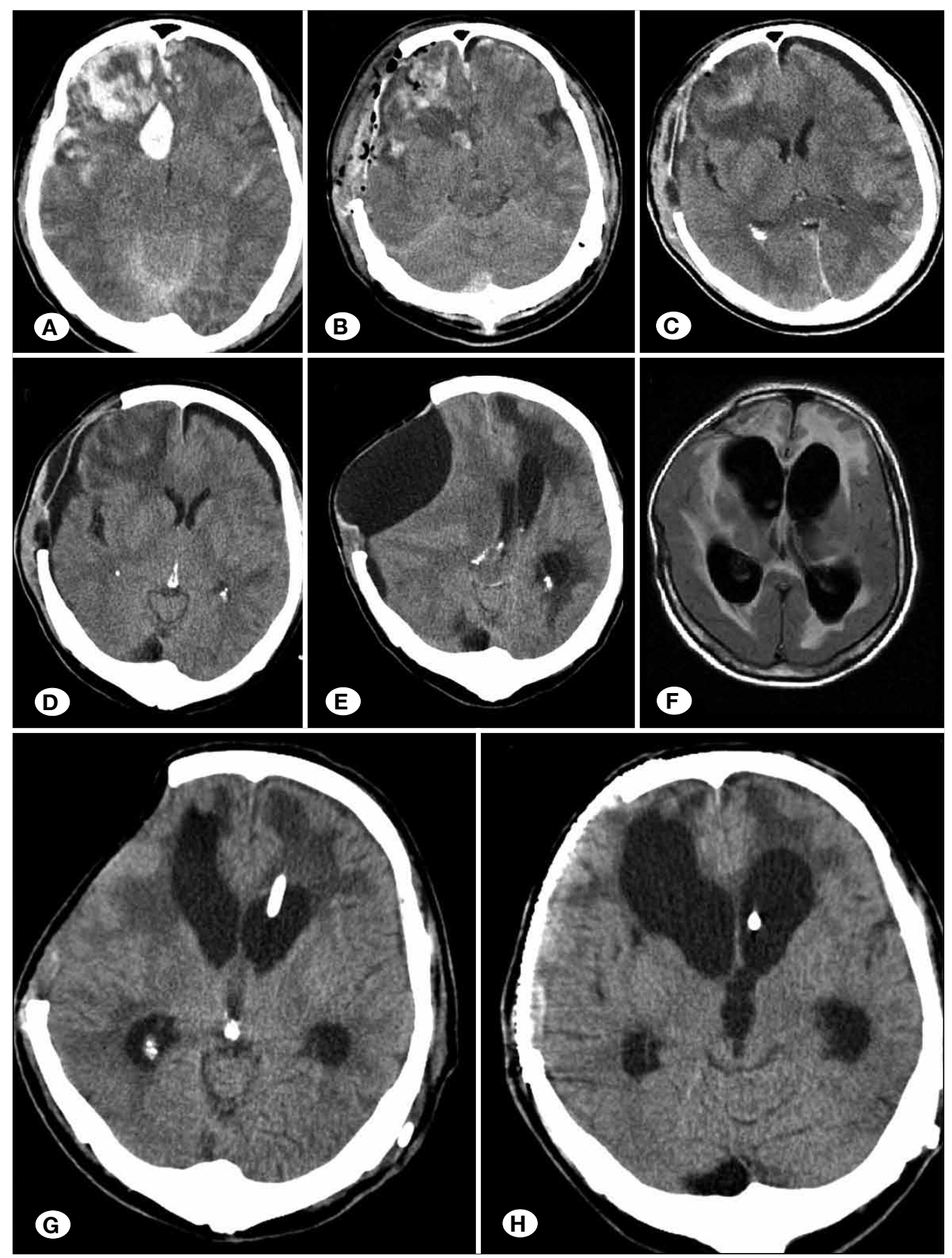

Figure 2: Patient 2 (shown in Table I) presented paradoxical herniation with sinking skin flaps in the post-operative period of 2 weeks to 2 months. Brain CT revealed a hemorrhagic contusion in the right frontotemporo-parietal lobe and diffuse brain swelling (A). CT images after emergency decompressive craniectomy (B). A head CT on POD 10 indicated a subdural hygroma in the left frontal region (C). The CT images on POD 30 exhibited an enlarged subdural hygroma in the left frontal region and a subgaleal fluid collection in the right frontal region and then the patient was mistakenly treated with continued lumbar CSF drainage (D). CT images on POD 56 revealed increased subgaleal fluid collection with flattening of overlying cortex and significant midline shift while the patient's neurologic status declined to a GCS of 4 (E). After removing the external lumbar drainage, being placed in the Trendelenburg position and receiving sufficient hydration intravenously, magnetic resonance imaging on POD 106 showed markedly reduced subdural hygroma, resolution of midline shift, and mild ventricular expansion on post-operative day 106 (F). On POD 122, the VP shunt for hydrocephalus was performed (G). CT images after cranioplasty (H). 

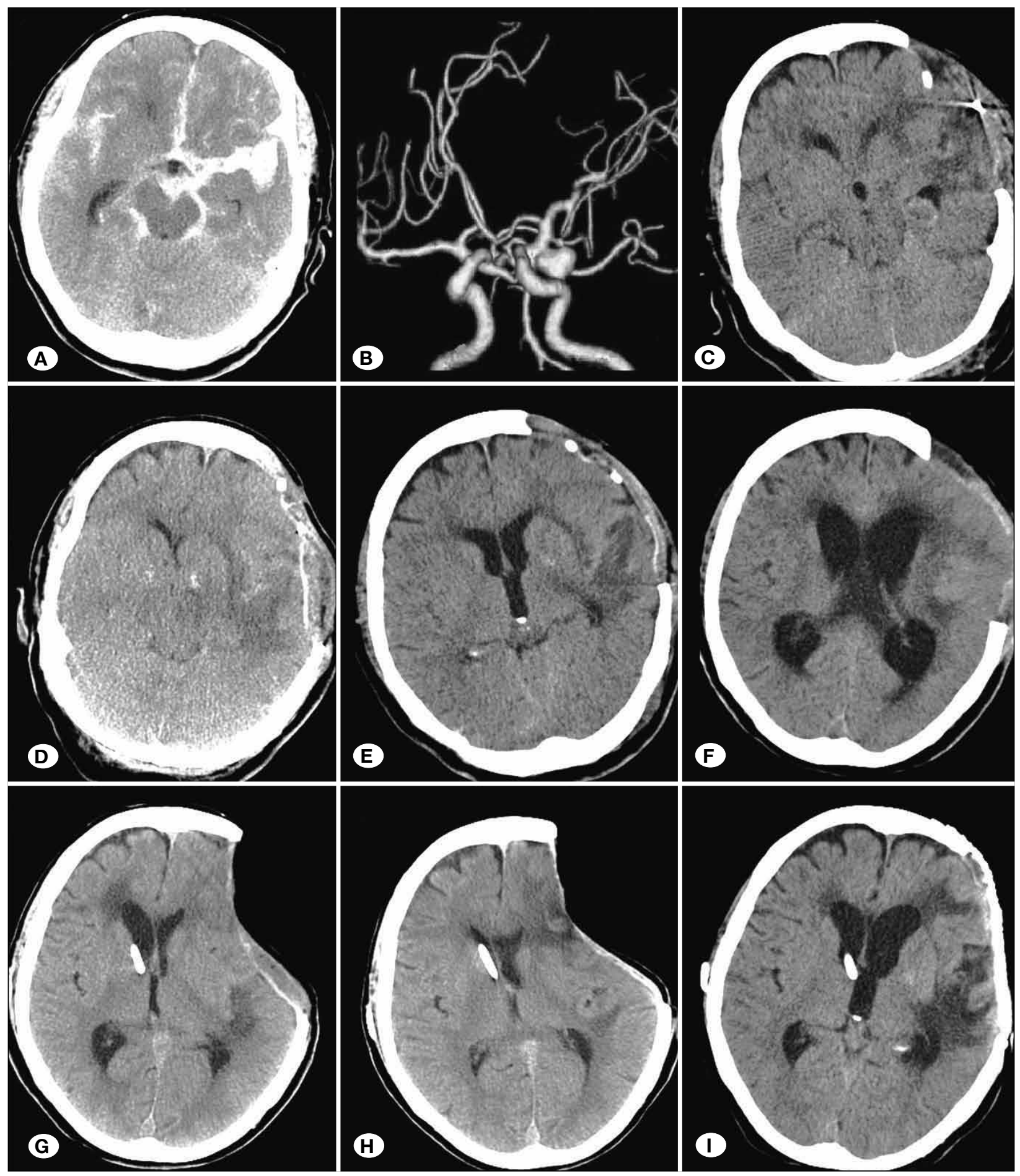

Figure 3: Patient 1 (shown in Table I) presented paradoxical herniation twice after unilateral decompressive craniectomy. CT images before operation exhibited subarachnoid hemorrhage (SAH), a hematoma in the left sylvian fissure, brain swelling without midline shift (A). Her CTA scan revealed an aneurysm in the terminal segment of the carotid artery (B). CT images after emergency decompressive craniectomy, evacuation of hematoma in the left sylvian fissure, clipping of the aneurysm, and placement of an epidural drain tube with normal initial ICP (C). On POD 16, her GCS declined to 5 while her blood pressure dropped to 81/38 $\mathrm{mmHg}$ with the ICP decreased less than $1 \mathrm{~cm} \mathrm{H} \mathrm{H}_{2} \mathrm{O}$ and the head CT showed marked herniation (D). After being placed in the Trendelenburg position and receiving sufficient hydration. The CT images on POD 23 revealed the reversion of the midline shift (E). The patient presented difficulty obeying commands on POD 33 and head CT indicated an enlarged ventricular system with external brain tamponade (F). After emergency VP shunt, a head CT on POD 44 showed much smaller ventricular system than that on POD 33 (G). She began to present worsen headache, neck pain, and dizziness on POD 62. The CT images showed paradoxical herniation (H). Her perfect outcome after cranioplasty was obtained (I). 


\section{Treatment}

Sufficient hydration with 2,500-4,000 ml fluids per day was given intravenously in 13 patients while the dehydration agents were terminated. Seven patients were placed in the Trendelenburg position. The sources of CSF loss were blocked by clamping all drains in 13 patients. Two patients with continued lumbar CSF drainage underwent a slow injection of 20 and $50 \mathrm{ml}$ saline through lumbar drainage tube respectively. One patient was treated by re-programming of a ventriculoperitoneal (VP) shunt to a $200-\mathrm{mm}$ valve setting. Subsequently, six patients underwent cranioplasty.

\section{a RESULTS}

As soon as the diagnosis of paradoxical herniation was made, seven patients were placed in the Trendelenburg position and given sufficient hydration within 1 hour. The effectiveness of such treatment was supported by the return of responsive pupils in three patients and re-expansion of the sinking skin flap in four patients. Two patients with continued lumbar CSF drainage underwent a slow injection of 20 and 50 milliliter saline respectively through the lumbar drainage tube and showed improvements in symptoms. Six patients remained in stable condition with marked improvement of 3-5 in Glasgow Coma Scale (GCS) score and resolution of midline shift in the subsequent brain CT scans.

\section{DISCUSSION}

Decompressive craniectomy is usually performed to deal with uncontrollable intracranial hypertension due to a variety of causes. The conversion of the skull into an "open box" may bring some risks. It disrupts the Monro-Kellie model and brings a new variable called the atmospheric pressure, which is the equivalent of 14.7 pounds/in ${ }^{2}$ or $1033 \mathrm{~cm} \mathrm{H}$ (1). The atmospheric pressure, altered CSF circulation (9, $12)$ and cerebral blood flow $(26,31)$ could cause intracranial hypotension, which results in syndrome of the trephined. It is supported by our ICP readings ranging from -3 to $3 \mathrm{~cm} \mathrm{H}$ and the variations of the blood pressure which are $72-81 / 35-$ $46 \mathrm{mmHg}$ in 3 patients with earlier paradoxical herniation. The combination of this low-pressure state and brain gravity effect can cause brain sag (1), which leads to eventual paradoxical herniation if there is no effective treatment to reverse the process (24).

The syndrome of the trephined and its symptoms were firstly described by Grant and Norcross (16) in 1939, although the term itself had been coined elsewhere from an unreferenced source. It is also known as the "sinking skin flap syndrome" because of the sinking skin flap overlying region of decompressive craniectomy occurred in the subacute phase (36). The syndrome of the trephined is not uncommon following craniectomy with an incidence of $10 \%$ in our cohort (13 in 131 patients with decompressive craniectomy), which is consistent with the incidence in other study results that ranged from $7 \%$ to $11 \%$ (29). The patients presented persistent or orthostatic headaches, dizziness, mood disturbances, and memory difficulties within weeks to months after craniectomy, which can be resolved rapidly by cranioplasty (32). However, cranioplasty occasionally improve epilepsy and psychiatric symptoms occurred after craniectomy (15). Even if cranioplasty improves most symptoms, some residual memory, irritability, and dyspraxia may persist (34). Interestingly, we found that paradoxical herniation presented sinking skin flap in the post-operative period between 2 weeks and 2 months. The most obvious sinking skin flap with a low tension occurred during 2 weeks postoperatively and it was possibly caused by the receding of herniated brain tissues in the subacute and chronic phases (1).

The low ICP herniation and CSF losses cause the major postoperative complications showed in our studies. They elevated the pressure gradient across the craniectomy defects $(1,9)$, which resulted in the intracranial structures shift to infratentorium and compression of brainstem at the foramen magnum along with brain gravity. As shown in Table I, 11 patients with large skull defect presented paradoxical herniation after unique CSF drainage (one patient with CSF rhinorrhea, ten patients receiving lumbar puncture). There are seven patients who underwent external ventriculostomy along with lumbar puncture or continued lumbar CSF drainage and four patients received VP shunts. All of them developed paradoxical herniation. The data in our study are consistent with other studies $(1,9,13,29,34)$. Other studies $(4,17,19,27,28)$ also showed the paradoxical herniation caused by prolonged CSF leakage through occult dural defects or intraoperative CSF drainage. The occult CSF leakage secondary to skull base fracture is usually considered as a primary cause of intracranial hypotension because of its difficult locating and frequent recurrence (23).

The patient with CSF depletion or intracranial hypotension presents headache, vision changes, nausea, neck stiffness, and photophobia. Unfortunately, these symptoms are noncharacteristic. They will lead to the misdiagnosis of meningitis and then undergoing lumbar puncture for CSF examination (22). A large sinking skin flap at the region of decompressive craniectomy reveals the need of further evaluation via diagnostic imaging. The subdural or epidural hygroma with expansion of the overlying skin flap could cover up the sinking defect (32). Hydrocephalus is another differential diagnosis with characteristic diagnostic imaging presented similar symptoms. However, hydrocephalus accompanied with low-compliance ventricles (e.g., slit ventricle syndrome) may confound the clinicians' diagnosis. The misdiagnosis will worsen the condition of the patient because of the different treatments. The compression of the subarachnoid space at the region of decompressive craniectomy secondary to the force of atmospheric pressure leads to the blockage of CSF circulation, which may cause the subdural or epidural hygroma, hydrocephalus and the subgaleal fluid collection (1). The condition of the patient with paradoxical herniation could be progressively worsening while the pressured brain tissue shifted along with its compressive strength on posterior fossa structures. Therefore, the treatments to revert the herniation are essential. The treatments are based on elevation of ICP and elimination of the pressure gradient across the craniectomy defects. It is also important to avoid VP shunts and external 
ventriculostomy. As paradoxical herniation progresses, the patients may present with alterations in consciousness, vision and pupillary changes, brainstem localizing signs, and autonomic instability. More remarkably, paradoxical herniation can occur with normal ICP values $(8,20)$.

Imaging plays important roles in the diagnosis of paradoxical herniation. Despite of limited emergency application, magnetic resonance imaging (MRI) is known as the best technique for demonstrating the characteristic pattern of diffuse dural gadolinium enhancement, which reveals intracranial hypotension. Focal and unilateral postsurgical enhancement is easily differentiated from the intracranial hypotension (21). Furthermore, imaging also reveals subdural fluid collections, crowding of posterior fossa by midline structures, shrinking of prepontine and perichiasmatic cisterns, congestion of cerebral venous sinuses, and enlargement of pituitary gland (5, 22). In addition, bilateral subdural hygromas with the lesions of thalamus, basal ganglia, cerebellum and brainstem, the signs of worse prognosis, are commonly supporting pieces of evidence $(5,18)$.

The treatment of paradoxical herniation is an emergency management in neurocritical care units based on eliminating the pressure gradient across the craniectomy defects, involving immediately placing the patient in the Trendelenburg position, providing IV hydration, and stopping all treatments of reducing ICP. Hyperosmolar solution administration, CSF drainage, elevation of bed height, and hyperventilation should be avoided $(5,9)$. Failure to recognize the shift from high to low ICP states can result in rapid deterioration of the patients because of the dramatically different treatment (9).

Moreover, the source of CSF loss should be blocked by clamping all drains and repairing the identified CSF fistula site without any delay. But even without lumbar puncture or CSF shunt, the patient with spontaneous or undiagnosed preexisting dural defects may develop intracranial hypotension (35). It is difficult to recognize these defects at the cervicothoracic junction and lumbar region (25) because of the limited imaging sign $(2,6)$. Intrathecal gadolinium-enhanced magnetic resonance cisternography has more advantage than CT myelography and nuclear medicine studies in detecting leaks in low and high-flow states (3). It is referred as the best modality in inspecting anatomic leaks (30).

Epidural blood patch has become the accepted treatment which relieves the clinical symptoms within hours $(9,10,30)$. Interestingly, the symptoms of two patients with continued lumbar CSF drainage were rapidly improved by undergoing a slow intrathecal injection of 20 and 50 milliliter saline through the lumbar drainage tube respectively. However, the effects of this treatment were temporal, which reveals the need of more detailed investigation.

Early cranioplasty, conversion of an "open box" to "closed box", is becoming increasingly adopted. It has been demonstrated that cranioplasty can improve cerebral blood flow and CSF circulation in the defect area (33). Additionally, cranioplasty is beneficial to the elevation of superior sagittal sinus pressure, cerebral metabolism, prevention of cerebral cortical thinning and improvement of cerebral vascular reserve capacities (11, 37). As shown in Figure 3A-I, a 68-year-old woman developed paradoxical herniation on the post-operative 5 day after clipping of ruptured middle cerebral arterial aneurysm on the left and decompressive craniectomy. She was improved rapidly after being placed in the Trendelenburg position and receiving sufficient hydration. She was discharged from the hospital due to lack of financial support for any further treatment including cranioplasty. She began to experience worse headaches, neck pain and dizziness 62 days postoperatively. She was diagnosed with paradoxical herniation with the support of imaging examination. Then she received cranioplasty and got some improvements. Her perfect outcome suggests that cranioplasty is a definitive treatment for paradoxical herniation.

\section{- CONCLUSION}

Paradoxical herniation caused by intracranial hypotension can rapidly progress especially along with spontaneous, traumatic, or iatrogenic CSF depletion. It is important for neurosurgeons to suspect paradoxical herniation in a subset of patients with large craniectomy defects and a tense skin flap without sinking during the post-operative 2 weeks, patients with sunken skin flap at the craniectomy sites, and patients presented new nonspecific neurologic complains of fever, headache, vision disturbance, autonomic irregularities, and altered consciousness in the postoperative period of 2 weeks to 2 months. Paradoxical herniation is rapidly reverted by improving CSF hydration and performing early cranioplasty. The misdiagnosis of paradoxical herniation can cause the rapid deterioration of patients, which reminds us to deal with this condition more carefully and seriously especially for the patient treated by non-neurosurgical clinicians initially.

\section{- REFERENCES}

1. Akins PT, Guppy KH: Sinking skin flaps, paradoxical herniation, and external brain tamponade: A review of decompressive craniectomy management. Neurocrit Care 9: 269-276, 2008

2. Akkawi N, Locatelli P, Borroni B: A complicated case of intracranial hypotension: Diagnostic and management strategies. Neurol Sci 27:63-66, 2006

3. Albayram S, Gunduz A, Saip S: Intrathecal gadoliniumenhanced MR-cisternography in spontaneous intracranial hypotension associated with Behçet's syndrome. Headache 47: 613-616, 2007

4. Atkinson JL, Weinshenker BG, Miller GM: Acquired Chiari I malformation secondary to spontaneous spinal cerebrospinal fluid leakage and chronic intracranial hypotension syndrome in seven cases. J Neurosurg 88: 237-242, 1998

5. Beck CE, Rizk NW, Kiger LT: Intracranial hypotension presenting with severe encephalopathy. Case report. J Neurosurg 89:470-473, 1998

6. Binder DK, Dillon WP, Fishman RA: Intrathecal saline infusion in the treatment of obtundation associated with spontaneous intracranial hypotension: Technical case report. Neurosurgery 51: 830-836, 2002 
7. Bliss M: Opening the closed box. In: Harvey Cushing-a life in surgery. New York: Oxford University Press, 2005:167-181

8. Bloch J, Regli L: Brain stem and cerebellar dysfunction after lumbar spinal fluid drainage: Case report. J Neurol Neurosurg Psychiatry 74:992-994, 2003

9. Choi JJ, Cirivello MJ, Neal CJ, Armonda RA: Paradoxical herniation in wartime penetrating brain injury with concomitant skull-base trauma. J Craniofac Surg 22: 2163-2167, 2011

10. Cousins MJ, Brazier D, Cook R: Intracranial hypotension caused by cervical cerebrospinal fluid leak: Treatment with epidural blood patch. Anesth Analg 98:1794-1797, 2004

11. Dujovny M, Agner C, Aviles A: Syndrome of the trephined: Theory and facts. Crit Rev Neurosurg 9: 271-278, 1999

12. Dujovny M, Fernandez P, Alperin N: Post-cranioplasty cerebrospinal fluid hydrodynamic changes: Magnetic resonance imaging quantitative analysis. Neurol Res 19: 311-316, 1997

13. Fields JD, Lansberg MG, Skirboll SL, Kurien PA, Wijman CA: "Para-doxical" transtentorial herniation due to CSF drainage in the presence of a hemicraniectomy. Neurology 67:15131514, 2006

14. Fodstad H, Love JA, Ekstedt J, Fridén H, Liliequist B: Effect of cranio-plasty on cerebrospinal fluid hydrodynamics in patients with the syndrome of the trephined. Acta Neurochir (Wien) 70: 21-30, 1984

15. Gardner W: Closure of defects of the skull with tantalum. Surg Gynecol Obstet 80: 303-312, 1945

16. Grant FC, Norcross NC: Repair of cranial defects by cranioplasty. Ann Surg 110:488-512, 1939

17. Guido LJ, Patterson RH Jr: Focal neurological deficits secondary to intraoperative CSF drainage: Successful resolution with an epidural blood patch. Report of two cases. J Neurosurg 45: 348-351, 1976

18. Hadizadeh DR, Kovacs A, Tschampa $H$ : Postsurgical intracranial hypotension: Diagnostic and prognostic imaging findings. AJNR Am J Neuroradiol 31:100-105, 2010

19. Inoue T, Cohen-Gadol AA, Krauss WE: Low-pressure headaches and spinal cord herniation. Case report. J Neurosurg 98 Suppl 1: 93-95, 2003

20. Komotar RJ, Mocco J, Ransom ER: Herniation secondary to critical postcraniotomy cerebrospinal fluid hypovolemia. Neurosurgery 57: 286-292, 2005

21. Mokri B: Posture-related headaches and pachymeningeal enhancement in CSF leaks from craniotomy site. Cephalalgia 21: 976-979, 2001

22. Mokri B: The Monro-Kellie hypothesis: Applications in CSF volume depletion. Neurology 56:1746-1748, 2001
23. Mortuaire G, Louis E, Pellerin P: Sphenoidal cerebrospinal fluid rhinorrhea: An original surgical approach. J Craniofac Surg 15: 458-463, 2004

24. Payner TD, Prenger E, Berger TS, Crone KR: Acquired Chiari malformations: Incidence, diagnosis, and management. Neurosurgery 34: 429-434, 1994

25. Peng PW: Intracranial hypotension with severe neurological symptoms resolved by epidural blood patch. Can J Neurol Sci 31: 569-571, 2004

26. Sakamoto S, Eguchi K, Kiura Y, Arita K, Kurisu K: CT perfusion imaging in the syndrome of the sinking skin flap before and after cranioplasty. Clin Neurol Neurosurg 108: 583-585, 2006

27. Samadani U, Huang JH, Baranov D: Intracranial hypotension after intraoperative lumbar cerebrospinal fluid drainage. Neurosurgery 52:148-151, 2003

28. Samii C, Mobius E, Weber W: Pseudo Chiari type I malformation secondary to cerebrospinal fluid leakage. J Neurol 246:162-164, 1999

29. Sarov M, Guichard JP, Chibarro S: Sinking skin flap syndrome and paradoxical herniation after hemicraniectomy for malignant hemispheric infarction. Stroke 41: 560-562, 2010

30. Schievink WI: Spontaneous spinal cerebrospinal fluid leaks and intracranial hypotension. JAMA 295:2286-2296, 2006

31. Schiffer J, Gur R, Nisim U, Pollak L: Symptomatic patients after craniectomy. Surg Neurol 47: 231-237, 1997

32. Stiver SI: Complications of decompressive craniectomy for traumatic brain injury. Neurosurg Focus 26: E7, 2009

33. Van Putten MC Jr, Yamada S: Alloplastic cranial implants made from computed tomographic scan-generated casts. J Prosthet Dent 68: 103-108, 1992

34. Vilela MD: Delayed paradoxical herniation after a decompressive craniectomy: Case report. Surg Neurol 69: 293-296, 2008

35. Vogel TW, Dlouhy BJ, Howard MA: Use of confirmatory imaging studies to illustrate adequate treatment of cerebrospinal fluid leak in spontaneous intracranial hypotension. J Neurosurg 113: 955-960, 2010

36. Yamaura A, Makino $\mathrm{H}$ : Neurological deficits in the presence of the sinking skin flap following decompressive craniectomy. Neurol Med Chir (Tokyo) 17: 43-53, 1997

37. Yoshida K, Furuse M, Izawa A: Dynamics of cerebral blood flow and metabolism in patients with cranioplasty as evaluated by $133 \mathrm{Xe}$ CT and 31P magnetic resonance spectroscopy. J Neurol Neurosurg Psychiatry 61:166-171, 1996 